Muséologies

Les cahiers d'études supérieures

muséologies

\title{
Les missions du musée : entre contemplation et éducation
}

\section{Julie Guiyot-Corteville}

Volume 3, numéro 2, printemps 2009

URI : https://id.erudit.org/iderudit/1033561ar

DOI : https://doi.org/10.7202/1033561ar

Aller au sommaire du numéro

Éditeur(s)

Association Québécoise de Promotion des Recherches Étudiantes en Muséologie (AQPREM)

ISSN

1718-5181 (imprimé)

1929-7815 (numérique)

Découvrir la revue

Citer cet article

Guiyot-Corteville, J. (2009). Les missions du musée : entre contemplation et éducation. Muséologies, 3(2), 48-63. https://doi.org/10.7202/1033561ar d'utilisation que vous pouvez consulter en ligne.

https://apropos.erudit.org/fr/usagers/politique-dutilisation/ 
Article trois

\section{Les missions du musée:}

\section{entre contemplation et éducation}

JULIE GUIYOT-CORTEVILLE, ETHNOLOGUE DE FORMATION ET CONSERVATEUR EN CHEF DU MUSÉE DE LA VILLE À SAINT-OUENTIN-EN-YVELINES, A NOTAMMENT TRAVAILLÉ À LA CONSTITUTION DE COLLECTIONS CONTEMPORAINES SUR LES MODES DE VIE URBAINS DES ANNÉES 1970 À NOS JOURS ET ÉLABORÉ DES DISPOSITIFS DE MÉDIATION SUR LES THEMES DE LA VILLE ET DU PATRIMOINE ARCHITECTURAL CONTEMPORAIN. CE TRAVAIL PIONNIER A PERMIS DINSTRUIRE AVEC SUCCĖS UN DOSSIER DE CANDIDATURE AU LABEL PAYS ET VILLE DART ET DHHISTOIRE, ATTRIBUÉ POUR LA PREMIERE FOISÀ UNE VILLE NOUVELLE DE LA SECONDE MOITIÉ DU XXE SIECCLE. PRÉSIDENTE DE LA FÉDÉRATION DES ECOMUSÉES ET DES MUSÉES DE SOCIÉTÉ DEPUIS 2003, ELLE EST AUSSI ADMINISTRATRICE DE LICOM.

[jutle.gułyot-corteville@agglo-sqy.fr] 


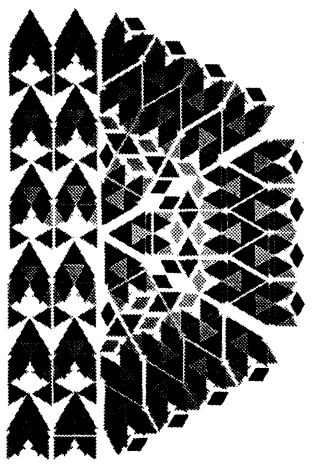

D'entrée de jeu, en tant que conservateur d'un musée de société qui traite d'une ville nouvelle et des modes de vie contemporains et en tant que présidente des écomusées et des musées de société, je dois dire que les termes de contemplation et d'éducation ne seraient pas forcément ceux que j'aurai cités spontanément pour définir nos missions. Sans doute, de mon point de vue, le premier renvoie-t-il trop à une position passive et le second à une posture par trop dirigiste ou volontaire. Je serai tentée de dire, non sans provocation, qu'ils évoquent pour moi spontanément tout ce à quoi nos musées tentent d'échapper dans l'évolution considérable qu'ils ont connue ces dernières années. Et je citerai volontiers Patrick Prado : «Ces musées seraient ceux du dérangement plus que de l'arrangement, du manque plutôt que du tropplein, de l'interrogation plutôt que du péremptoire, de l'intranquillité plutôt que de la quiétude, de la fragilité plutôt que de l'incassable: des musées de la fragilité des hommes ${ }^{[1]}$." Il y a peu de place dans cette approche pour la contemplation et l'éducation!

Mais comme l'intitulé de ce séminaire ${ }^{[2]}$ introduit le terme complémentaire du " entre ", de l'intermédiaire, de l'un et de l'autre..., il laisse de fait une grande latitude de pensée et de proposition. Ce texte s'évertuera donc à définir ce qui peut exister entre l'un et l'autre, entre la contemplation et l'éducation; entre ces deux garde-fous qui sont aussi les missions " historiques " qui ont fondé la légitimité des musées, missions régaliennes que sont venus bousculer Georges-Henri Rivière ${ }^{[3]}$ et surtout Hugues de Varine ${ }^{[4]}$ dans la construction du concept d'écomusée dans les années 1970, substituant le patrimoine aux collections, le territoire au musée et la population aux visiteurs. Philippe Mairot ${ }^{[61}, 30$ ans plus tard, constate que ces musées, belles demeures d'éternité qui abritent des objets de peu de valeur 
PRADO, Pat

Territoire de l'objet, faut-il fermer les musées. Paris: Éditions des archives contemporaines, 2003.

[2]

Le musée, entre contemplation et éducation, nouvelles tendances.

[3]

WEIS, Henri.

La muséologie selon Georges Henri Rivière. Paris: Dunod,

1989.

[4]

DE VARINE, Hugues. L'initiative communautaire, recherches et expérimentations. Macon: Éditions W; Savigny-le-Temple: MNES,

1991.

[5]

MAIROT, Philippe

Dépanthénéisons les musées. 2007 (document non publié). marchande, ont été transformés de l'intérieur, dans leurs missions, dans leurs métiers et jusque dans leurs modalités d'évaluation, car il n'y règne plus seulement le beau souci de la délectation esthétique autour du bien, mais s'y est développée une attention vive, en lien avec la population dont ils gardent le patrimoine, une interrogation incessante et critique de l'héritage, le réexamen perpétuel des questions d'identité et de complexité.

Ces musées de société, que l'on a parfois qualifiés de fossoyeurs de mémoire et d'identité, ont, dans la seconde moitié du XXe siècle, accompagné notre société dans ses changements les plus douloureux, "penseurs » de la société et de ses plaies (exode rural, désindustrialisation...); pour cela, ils ont été tour à tour remerciés d'avoir sauvegardé la mémoire et inculpés de l'avoir transformée en nostalgie d'un temps révolu. Véritable observatoire du changement et parfois du chaos qui pouvait s'ensuivre, ils ont distillé du sens et posé des bornes comme des points lumineux pour se repérer dans un espace social en recomposition. Pas de place dans ce paysage chaotique pour la contemplation.

Il m'arrive en tant que visiteur de musée de contempler et bien souvent d'apprendre. Alors joue-t-on sur les mots ici? Au fond, je ne le crois pas, car toute la logique de l'expérience que je vais tenter de relater s'appuie sur la notion de participation, pierre angulaire des écomusées, d'action, de questionnement, ce que peut recouvrir aujourd'hui le terme de médiation. Je suis convaincue que le principal acquis de ces dernières années par le biais des nouvelles politiques des publics, et où il nous reste encore beaucoup de travail, c'est l'instauration d'un dialogue, encore balbutiant, entre les équipes des musées et les publics. Cela implique un changement radical de posture très difficile à surmonter pour les professionnels que nous sommes, notamment d'une circulation verticale, de haut en bas, des connaissances qui sont les nôtres vers un public à instruire. Refusant d'être généralement ce qu'on lui demande, un lieu de certitudes, d'enseignement, le musée de société doit jouer sur le registre du questionnement, de l'interrogation, en combinant sens intrinsèque, sens réfléchi et sens inculqué.

On l'aura compris dans cette introduction, l'expérience du musée de la Ville, qui s'inspire grandement des musées de société, invite plus à révéler qu'à contempler, à questionner qu'à éduquer, à décoller le regard du quotidien qu'à se « délecter », car le chef-d'œuvre ici ne peut être qu'une trouvaille révélée par les habitants eux-mêmes dans la confrontation de leurs jugements de valeur, de leurs points de vue avec les propositions et les connaissances du musée. Comme nous le verrons par la suite, l'objet «non identifié» du musée, la ville nouvelle sur un territoire émergeant, sans légitimité historique, nous obligeait à construire ce dialogue pour aller à la rencontre de nos publics qui ne seraient guère venus spontanément au musée. 


\section{Un musée pour donner du sens à un territoire nouveau}

Le musée de la ville de Saint-Quentin-en-Yvelines a pour mission d'enregistrer les mutations successives d'un territoire qui est passé en moins de 20 ans d'un vaste plateau agricole à une agglomération de 150000 habitants regroupant sept communes. Il tente d'analyser les enjeux urbains et politiques des villes nouvelles tout en menant une réflexion sur les modes de vie urbains et contemporains.

Dans ce contexte, il est proche des musées de ville dans son questionnement et sa démarche. Néanmoins, il doit réfléchir différemment sur la notion de patrimoine. Dans une ville traditionnelle, les objets patrimoniaux puisent leur légitimité dans plusieurs siècles d'histoire. À Saint-Quentin-enYvelines, l'approche patrimoniale du musée est liée à un concept : le champ d'expériences sociales, politiques et urbanistiques des villes nouvelles. Et c'est à partir de l'analyse des logiques qui ont présidé à la constitution du territoire que le musée décline l'inventaire urbain: plaquettes de promoteur, bâtiments caractéristiques d'un courant architectural, d'un projet social, d'une époque, œuvres d'art public, maquettes, supports de communication institutionnelle...

Le musée est donc à la fois un lieu de recherche et un lieu de collecte, deux activités indissociables : l'intérêt de l'objet est lié principalement à l'histoire qui s'y rapporte. La recherche, travail de terrain, d'observation et d'entretiens auprès des acteurs de la ville, est menée en partenariat avec des organismes scientifiques: c'est de la confrontation des différents discours, celui des aménageurs, des architectes, des habitants, des élus, que peut naître une compréhension de ces phénomènes urbains. Les archives orales et audiovisuelles permettent d'animer les plans et les maquettes derrière lesquels se cachent bien souvent des convictions, des passions, des conflits, bref, de multiples histoires de vie.

La recherche pluridisciplinaire et les collections sont les principaux outils pour comprendre le tissu urbain si singulier des villes nouvelles, ni «banlieusard " ni « traditionnel». Le musée développe avec les habitants cette approche critique de leur environnement et de leur cadre de vie. La cohésion urbaine telle que nous l'entendons ne va pas dans le sens de la construction d'une identité saint-quentinoise encore abstraite pour la plupart des habitants dont le premier référent reste la commune. Il s'agit plutôt ici de permettre aux habitants de se situer dans la ville nouvelle et au sein de l'aventure collective qu'a représentée sa construction en moins d'une génération. 
DAVALLON, Jean.

"Les objets ethnologiques peuvent-ils devenir des objets de patrimoine? ". In. GONSETH, Marc-Olivier, Jacques HAINART et Roland KAEHR. Le musée cannibale. Neuchâtel, Suisse: Musée d'ethnographie, 2002.

[7] Id., p. 270.

[8]

Extraits des propos de Nathalie $\mathrm{HEINICH}$, colloque sur le patrimoine culturel immatériel de l'Europe: inventer son inventaire. Colloque de l'Institut national du Patrimoine, Paris, 27 novembre 2007.
Permettre à une population de s'approprier l'histoire d'un territoire qui s'est modelée à coups de lois, de décrets et de tâtonnements empiriques, à la recherche d'un nouveau modèle urbain et de nouveaux modes de vie, n'est pas aisé, car les habitants eux-mêmes n'en ressentent nul besoin et ne voient ici nul patrimoine. Le rapprochement entre ville nouvelle et patrimoine ne va pas de soi pour les visiteurs. A fortiori, une ville nouvelle d'art et d'histoire ne manquera pas d'engager questionnement et étonnement. Je tenterai donc ici de montrer comment cette notion de patrimoine s'est construite à partir d'une vision dynamique du territoire, en croisant et en confrontant regards et discours de différentes catégories d'acteurs: aménageurs, élus, architectes, habitants. Le musée de la ville, anciennement écomusée de Saint-Quentinen-Yvelines, cheville ouvrière de cette initiative, s'appuiera sur le concept de «trouvaille » développé par Jean Davallon dans Le Musée cannibale ${ }^{[6]}$ pour faire surgir une légitimité patrimoniale là où il n'y avait jusque-là qu'un territoire en devenir souvent considéré comme sans histoire, ni mémoire:

La chose qui fait patrimoine doit être une trouvaille; trouvaille concrète comme en archéologie où personne ne soupçonne l'existence de l'objet avant de le mettre à jour. Trouvaille en termes de valeur lorsqu'une chose (un édifice, un morceau de ville, un savoir...) qui était totalement délaissée apparaît soudainement avoir une valeur inestimable. Si cette trouvaille n'apparaissait pas auparavant, c'est que la chose était dans une situation qui interdisait de la voir ou encore que le regard qu'on portait sur elle ne permettait pas de la percevoir ${ }^{[7]}$.

On s'interrogera sur l'« urgence " relative d'ériger en patrimoine un gisement de ville si contemporain, sur son utilité politique et sociale. On peut également questionner la polysémie croissante de la notion de patrimoine qui ne se réduit plus au monument, mais embrasse toutes les fonctions de la ville. On peut encore se demander : Est-ce que la protection la plus efficace passe par l'appropriation des lieux par les habitants? Où se situe le patrimoine entre le mouvant (l'immatériel) et le fixe (le matériel) ? Est-il utile de fixer ce qui par définition échappe à la fixation? Ne risque t'on pas à terme de détruire ou d'user l'objet, bref de banaliser la " trouvaille " ${ }^{[8]}$ ?

En s'arrimant à une démarche scientifique, le musée va élaborer un certain nombre de critères qui expliciteront ce qui fait patrimoine dans la ville de son point de vue : le critère du temps et de l'histoire (ce qui fera date...), ce qui fait sens au regard de l'évolution du territoire et de ses enjeux, ce qui s'inscrit dans la mémoire collective. Mais toute la difficulté de ce travail réside dans la traduction de ces critères pour les différentes catégories d'acteurs qui font la ville. Contrairement au patrimoine légitimé par le temps, il existe autant de points de vue que d'acteurs et la question patrimoniale est encore souvent résolue à coups de jugements de valeur : «C'est beau, c'est laid, c'est réussi, c'est raté. » L'approche du patrimoine ici est largement déconnectée 


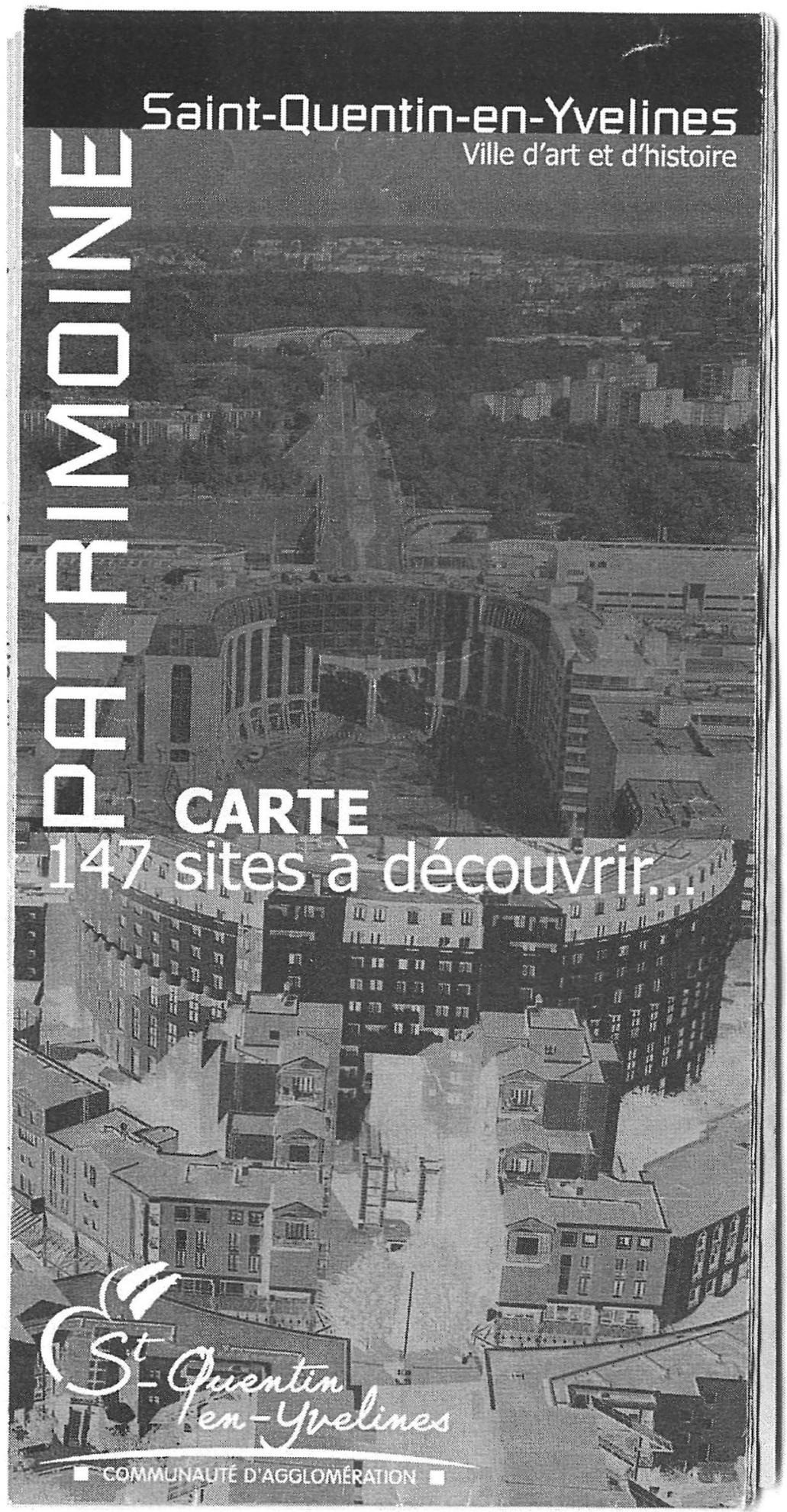

Carte du Patrimoine VAH Saint-Quentin-en-Yvelines. 
du classement ou de l'inventaire. Le patrimoine ne peut pas figer un espace urbain encore en devenir, même si nous avons conscience que la période des années 1970, genèse du territoire, est particulièrement fragilisée actuellement par un rejet des élus comme des habitants.

Il ne s'agit donc pas de passer "en force» notre vision savante du patrimoine en nous réfugiant derrière des critères scientifiques maîtrisés, mais d'engager un exigeant processus de médiation, en suscitant notamment les points de vue de nos interlocuteurs à partir desquels un dialogue peut s'engager. En amont du label Pays et ville d'art et d'histoire, cette médiation a été incarnée par un vaste dispositif de sensibilisation et d'échanges mené sur plusieurs années, de 2000 à 2006 (mais qui se poursuit évidemment). L'équipe du musée a rencontré le maire de chacune des sept communes, les anciens aménageurs de l'ÉPA (Établissement public d'aménagement), les architectes et de nombreux habitants pour échanger sur leur définition du patrimoine en général et sur celle de la ville nouvelle en particulier. Nous avons vite, au cours de ces échanges, délaissé ce terme, pour nous engager dans une pluralité de notions toutes constitutives de ce concept dans une approche urbaine et contemporaine, du cadre de vie au paysage, du développement économique à la mémoire familiale, des trajectoires quotidiennes aux visites spontanées réalisées par les habitants pour leurs hôtes de passage.

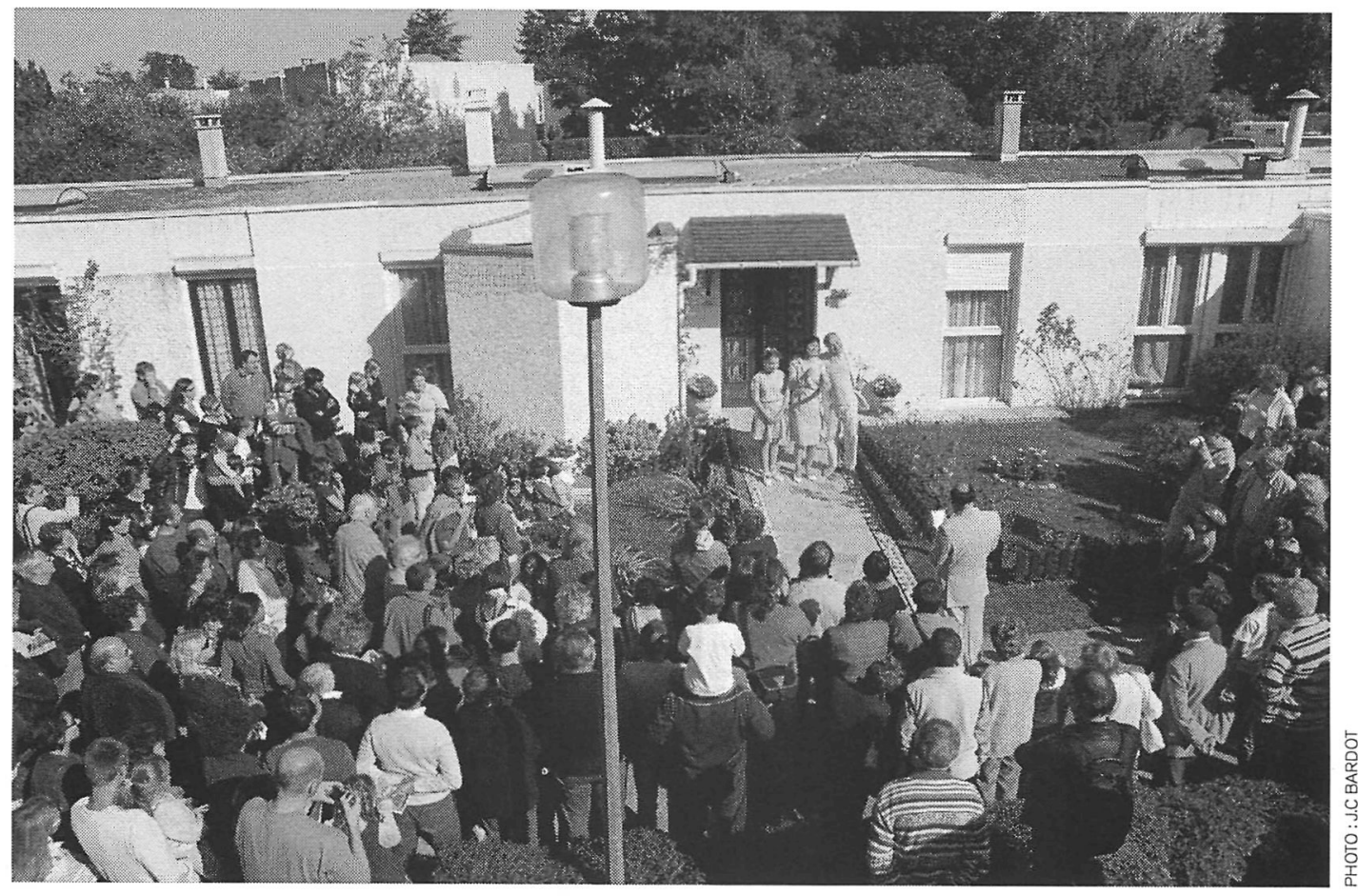


Si peu d'interlocuteurs cernaient ou reconnaissaient l'existence d'un patrimoine dans son approche la plus classique, tous témoignaient d'un attachement, d'une curiosité pour leur environnement. Des anniversaires de lotissement ou d'équipement en passant par des souvenirs biographiques attachés aux lieux, de nombreux indices montraient qu'un processus d'appropriation, en lien avec l'histoire singulière de la ville nouvelle, était bien à l'œuvre ici. La conscience d'avoir participé à une aventure exceptionnelle, la construction d'une ville en une génération, était opérante, toutes catégories d'acteurs confondues. Les élus du territoire, eux-mêmes dubitatifs quant à l'obtention d'un label d'art et d'histoire en 2000 lorsque François Barré, alors directeur de la Direction de l'architecture et du patrimoine architectural, les avait invités à postuler, sont aujourd'hui les premiers à revendiquer un processus de reconnaissance symbolique qui érige, au titre de "monument ", des constructions très contemporaines sur lesquelles ils pouvaient, quelques années auparavant, porter un jugement mitigé ou sévère. La carte du patrimoine éditée en 2006 devient un " manifeste » du label Ville d'art et d'histoire en réunissant toutes les typologies patrimoniales (architecture religieuse, rurale, ouvrière, contemporaine, logements, équipements, art public, parcs, etc.), toutes périodes confondues, dont une mosquée de 2005 et un foyer Sonacotra (Société nationale de construction de logements pour les travailleurs) de 2007. Elle vise à déplacer les frontières habituelles du patrimoine bâti en incluant plus largement le patrimoine immatériel illustré en partie par la mémoire collective et la vie des hommes qui façonnent ce territoire.

De ce point de vue, le label a constitué, sans nul doute, un des premiers consensus culturels au sein de la difficile intercommunalité. Si les aménageurs de l'ancien ÉPA ont immédiatement saisi cette démarche comme étant la reconnaissance de leur investissement sur le territoire, les architectes directement concernés prenaient souvent leurs distances avec un travail qu'ils ne revendiquaient parfois que modérément au sein de trajectoires qui les ont conduits ensuite ailleurs. Cette distance semblait à la mesure de l'espoir sans doute inconsidéré qu'ils avaient placé dans cette participation collective à un nouveau territoire guidé par l'innovation et l'utopie. Les entretiens montraient, pour autant, que ces expériences avaient profondément marqué leur réflexion. Le patrimoine des $\mathrm{XX}^{\mathrm{e}}$ et $\mathrm{XXI}{ }^{\mathrm{e}}$ siècles oblige à une construction collective qui intègre les témoins, ce qui constitue une chance et une contrainte. 
C'est donc bien de la confrontation de ces points de vue et de leur analyse pluridisciplinaire que s'est nourri le musée pour baliser et étayer son approche patrimoniale de la ville qu'il conçoit comme une construction collective forcément évolutive. C'est en cela avant tout un témoignage de notre époque, une vision du monde qui ne se résume surtout pas à un consensus, mais à un dialogue dont les conclusions ne nous appartiennent pas. Cette construction "polyphonique» du patrimoine, qui constitue déjà en soi un vaste dispositif de valorisation et de médiation, contraint et oriente les partis pris muséographiques et le dispositif d'action culturelle lui-même.

Entre contemplation et éducation, ily a bien un champ infini de pratiques développées par les musés. Mais les « recettes " professionnelles ne sont évidemment jamais transposables en l'état. Si ce qu'on peut observer chez nos collègues nourrit toujours la réflexion, la cohérence d'un projet global est indispensable. Cette cohérence ne se construit qu'avec tous les paramètres du projet: son objet, son environnement, ses objectifs. Et je dois dire, en tant que conservateur de musée de société, que les musées de beaux-arts, et notamment d'art contemporain, alimentent beaucoup la réflexion. Je suis en effet aujourd'hui convaincue qu'il est nécessaire de faire bouger les lignes et de bousculer les frontières. Les familles de musée sont le produit d'une histoire institutionnelle, mais les publics peuvent légitimement attendre du musée des approches plus transversales et plus poreuses où des questions de société pourraient aussi côtoyer des approches plastiques.

Afin de rendre plus concret mon propos je développerai trois axes qui semblent essentiels à notre projet. Les partis pris muséographiques des expositions, le principe de médiation qui structure les actions culturelles et les événementiels qui, chez nous comme dans beaucoup de musées, prennent une place de plus en plus importante et soulèvent beaucoup de questions.

La muséographie, pédagogie du questionnement?

La muséographie est une écriture qui signe l'identité du musée et, malgré son renouvellement en fonction des thèmes des expositions temporaires, certains principes intangibles participent de l'image du musée: décalage, questionnement, interpellation du visiteur, hiérarchisation des niveaux de lecture. D'ailleurs, ce principe d'interpellation du visiteur vient rompre tout principe de contemplation, même si la muséographie joue sur l'émotion, la magie des lumières et des installations et s'appuie sur le dépaysement. Il faut que le visiteur, après avoir pénétré dans l'espace, se sente transposé dans un autre univers qui constitue une première forme d'interpellation. Ce parti pris sensible peut échouer bien évidemment si le visiteur se sent exclu du propos parce qu'il reste extérieur au dispositif ou le rejette. 

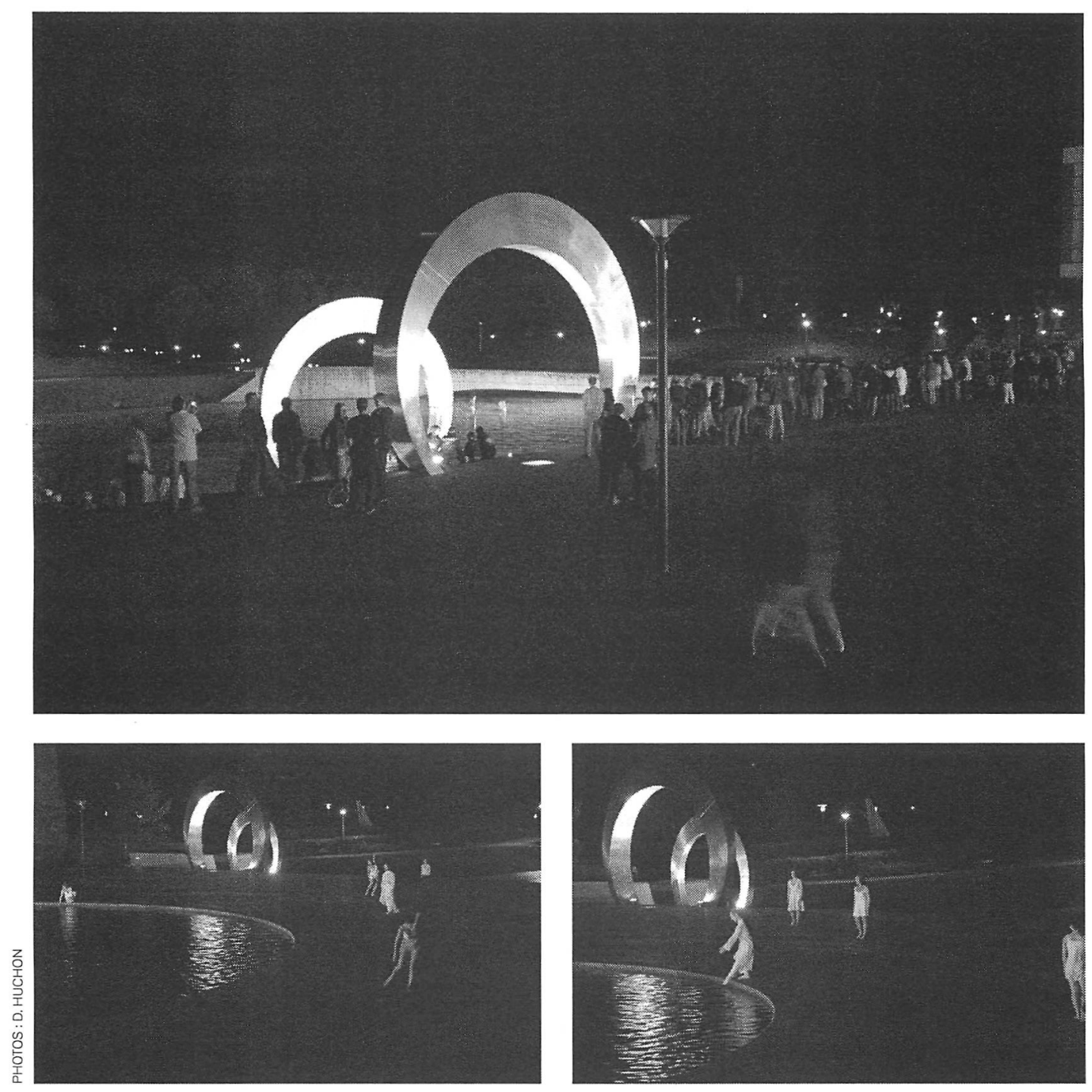

Nuit des musées, 17.05.2008, sculpture Marta Pan, compagnie La Folia-Cristine Bastin, coll. MDV2, MDV4, MDV9. 


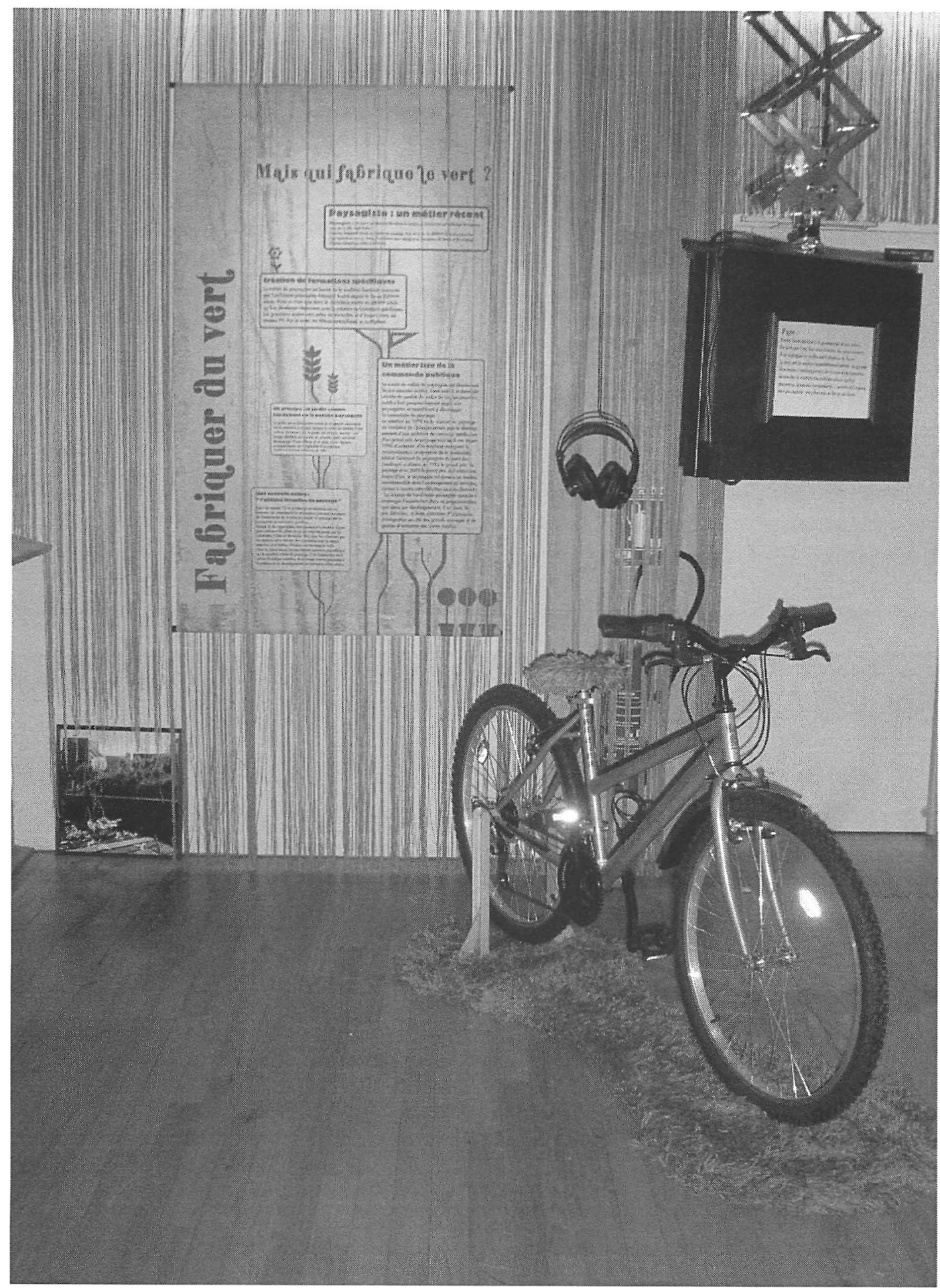


De même que l'arrivée massive d'objets usuels de la vie quotidienne a obligé les musées de société, dans les années 1970, à renouveler l'approche muséographique du chef-d'œuvre (reconstitution de lieux), scénographier le contemporain oblige à inventer une nouvelle contextualisation dans l'espace. Dès qu'il s'échappe de l'histoire révolue, le musée prend son auditoire à contrepied (le public apprécie particulièrement la nostalgie...), mais c'est bien là, dans cet espace social qu'il n'a pas l'habitude d'occuper, qu'il peut aussi trouver les conditions de son renouvellement et les prémisses d'une nouvelle définition de ses missions. Les muséographies s'appuient dès lors sur des contrastes et des distances symboliques qui permettent de mettre en perspective l'objet déjà en collection et pourtant encore dans la cuisine des visiteurs, ce qui ne manque pas de les déconcerter et d'interroger l'idée qu'ils se font du musée comme témoin d'une époque révolue.

Présenter une essoreuse à salade comme une porcelaine de Sèvres peut avoir du sens en questionnant le visiteur sur le statut de l'objet et son rapport au temps. Ce n'est certes pas une révolution et Jacques Hainard, longtemps conservateur du musée d'ethnologie de Neûchatel, a beaucoup innové dans ce domaine, en bousculant les codes muséographiques. Mais rien n'est plus décevant que de voir, dans certains musées, des séries d'objets qui se concluent par des objets contemporains comme objets prétextes pour inclure cette dimension, sans pour autant la traiter. Les scénographies élaborées en étroite collaboration avec les équipes de scénographes forment un tout avec le propos du musée, comme un métalangage qui interpelle et illustre, de façon à la fois drôle, poétique et provocante, le questionnement du musée. L'exposition est un tout composé comme un parcours rythmé de contrastes. Elle est conçue pour surprendre, faire sourire, jouer sur les décalages chronologiques tout en puisant dans les références partagées dans la mémoire collective, s'appuyer sur des symboles, retrouver le vocabulaire classique du musée pour mieux le détourner. Ces dispositifs réussissent, ou pas, et leur évaluation n'est pas toujours aisée. Car, dans toute exposition, la prise de risque existe et elle réside dans cet équilibre subtil entre le dépaysement recherché et des signes partagés, entre la provocation et l'humour distillé pour traiter de questions qui font débat. Je pense à ces assiettes sérigraphiées avec les monuments que l'on trouve dans les hauts lieux patrimoniaux et touristiques, que nous avions remplacés par des tours, des barres et des pavillons de banlieue. Ici la scénographie, si elle tient compte de l'objet, n'est pas au service de la collection mais du propos. Et cela me semble infiniment plus complexe. Pour une exposition sur les parcs et les jardins qui s'élevaient contre l'idée reçue selon laquelle les villes nouvelles avaient "bétonné» la campagne, la scénographie avait utilisé le gazon synthétique pour renforcer lidée de la nature comme une construction de l'homme. Nous ne sommes pas dans le registre de la contemplation, mais bien dans celui d'une ambiance, 
d'une émotion, d'un esprit des lieux radicalement différents à chaque fois qui projettent le visiteur dans un univers qu'il s'approprie ou pas et dans lequel il est directement questionné par rapport à ses propres références, en s'attaquant parfois à ses convictions.

\section{La médiation, un dialogue à construire?}

Le dispositif d'action culturelle repose, quant à lui, sur la transmission intergénérationnelle, permettant un dialogue et un accompagnement entre enfants et parents ou grands-parents. On observe ainsi que les dispositifs pédagogiques permettent aussi aux adultes " décomplexés» d'intégrer les contenus. Le principe central qui guide l'ensemble des actions, qu'elles s'adressent à un public scolaire ou aux visiteurs libres, s'articule autour de

[9]

CAILLET, Élisabeth.

À l'approche du musée,

la médiation culturelle. Lyon: Presses universitaires de Lyon, 1995.

[10]

BORDEAUX, Marie-Christine. Les enjeux de la médiation culturelle. In. Publication des actes de Musées et société aujourd'hui. Musée Dauphinois de Grenoble, 2008, p. 188-194. la médiation, c'est-à-dire s'appuyer sur les connaissances et les positions des visiteurs pour ensuite tisser un dialogue. Sur les questions d'art public, d'architecture contemporaine par exemple, nous sommes presque systématiquement confrontés à des jugements de valeurs et à des critiques souvent virulentes. C'est la matière à partir de laquelle nous travaillons pour déconstruire progressivement ces jugements ou du moins les mettre en perspective, introduire de la distance, établir des comparaisons. Peut-on alors parler d'éducation au patrimoine? Oui, dans le sens où l'on introduit des connaissances; non dans le sens où l'on n'impose pas un savoir légitime lié à notre position de "sachant ", mais où l'on échange des arguments sans donner pour autant une seule réponse. On introduit toujours de la complexité qui déstabilise le jugement sans le condamner ou le rejeter, ce que je qualifierais d'approche "compréhensive" au sens sociologique du terme. Les ateliers scolaires fonctionnent sur le même modèle avec des mots spontanément “jetés " par les enfants pour décrire la ville. Les termes sont au départ souvent négatifs : pollution, béton... Les enfants travaillent ensuite à partir de maquettes et d'images sur une ville idéale que l'on compare, à la fin de l'atelier, à leur propre environnement. Ils sont alors souvent surpris des similitudes entre le modèle idéal et leur lieu de vie. Si jinsiste sur cette question, c'est que le terme de médiation est employé presque systématiquement aujourd'hui dans les musées pour qualifier l'action culturelle, sans pour autant que ses principes essentiels soient appréhendés, notamment celui de construire un dialogue à partir des connaissances respectives des interlocuteurs. Sur cette question je renvoie le lecteur aux écrits d'Élisabeth Caillet ${ }^{[9]}$ ou de Marie-Christine Bordeaux ${ }^{[10]}$. 


\section{L'événementiel : vers une démocratisation des connaissances?}

Je terminerai par le champ de l'événementiel qui, de prime abord, pourrait se rapprocher davantage de la contemplation collective. S'accrocher notamment aux évènements nationaux, comme les Journées du patrimoine ou la Nuit des musées, est un moyen de toucher un public plus large, qui ne viendrait pas forcément au musée. C'est pour les habitants, avant tout, un moment convivial et festif, libéré de l'entrave des codes culturels et sociaux du musée. Le musée peut alors prendre appui sur ces évènements pour susciter la curiosité et décoller le regard du paysage urbain quotidien dans lequel les habitants ne décèlent nul patrimoine. Le principe que nous développons systématiquement pour animer les lieux s'inscrit dans la pluralité des formes artistiques et des patrimoines. Depuis trois ans maintenant, les visites des journées du patrimoine sont théâtralisées par la compagnie Thalia-Théâtre, en résidence au musée. Soit la compagnie reprend des textes du patrimoine littéraire peu connus, soit elle rédige des textes en introduisant de la fiction à partir des récits d'habitants ou d'acteurs de la ville collectés par le musée. La Nuit des musées décline depuis trois ans des spectacles de chorégraphie contemporaine en lien avec les œuvres d'art public dans la ville. On pourrait penser que nous multiplions les difficultés en rajoutant à du patrimoine "non identifié» des textes difficiles ou de la chorégraphie contemporaine peu comprise généralement du grand public... Curieusement, la proposition séduit le public qui accepte d'être bousculé dans la mesure où cela se produit sur un temps de rupture, un accroc dans le quotidien, un accident dans l'espace public qui fera sans doute qu'on ne percevra plus le lieu de la même façon après cette transgression dans le temps et l'espace. L'interprétation de l'artiste paraît rassurante et peut se substituer à sa propre interprétation. Lélément patrimonial mis en lumière devient alors un lieu d'interrogation, consensuel ou pas. Le fait que des artistes - qu'ils soient écrivain, danseur, plasticien... - se saisissent de cette matière modifie notre propre vision du patrimoine avec une plus-value « sensible». Les artistes se saisissent donc d'une matière concrète qu'ils transforment en élément subjectif et impalpable. Ils mettent du jeu dans les différents éléments du puzzle, l'espace public, l'œuvre, l'architecture, le paysage... Cette mécanique introduite entre les pièces crée le mouvement. Outre les moyens et le temps que ces manifestations exigent, souvent au détriment des autres missions du musée, cela permet au musée de rendre visibles un territoire, un patrimoine, une œuvre ou une architecture jusque-là transparents. J'évoquerai le terme de ravissement plutôt que de contemplation, ravissement au sens où l'évènement nous ravit à nous-mêmes, un moment où l'on est absent et totalement là, dans des champs qu'on explore peu d'habitude. 
En conclusion, je dirai que, de la contemplation à l'éducation, le musée bénéficie aujourd'hui d'un champ d'intervention presque sans limite, si ce ne sont les moyens financiers et les orientations politiques. Il me semble en tous les cas important d'expérimenter sans relâche, d'évaluer le plus possible pour se remettre en question et recommencer à nouveau. Le propre de petites institutions comme le musée de la ville à Saint-Quentin-en-Yvelines est que nous n'avons aucune autre marge de manœuvre que celle d'expérimenter sans cesse, sous peine de disparaître, car le moins que l'on puisse dire c'est que notre sujet, comme notre positionnement, nous obligent en permanence à séduire, à surprendre.

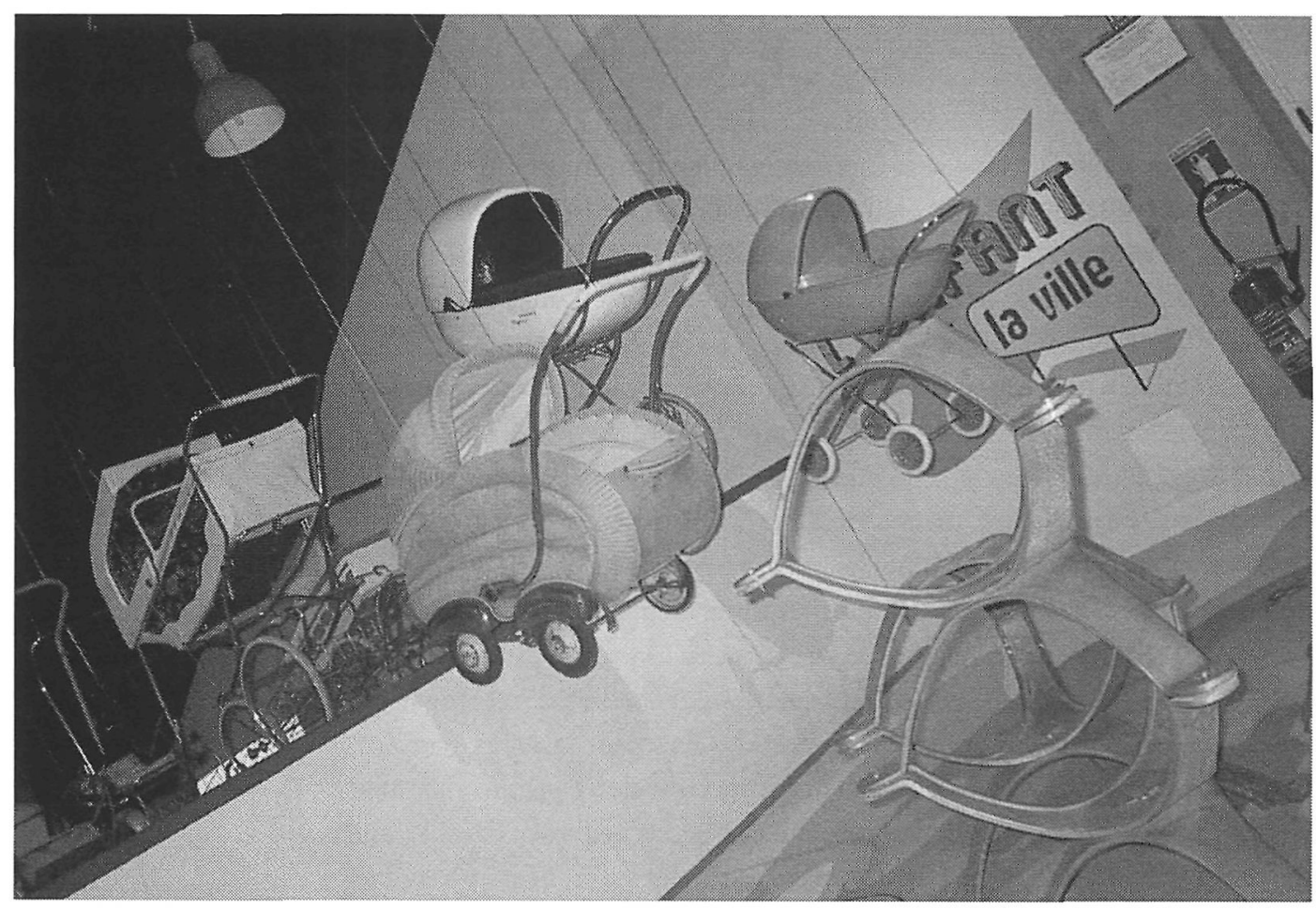




\section{Summary}

[Translated by Micheline Giroux-Aubin]

This article questions the missions expected of museums, which are included in terms such as delight in the presence of exceptional works and education through a conventional educational approach. These museum's collections, originating in the seventies, are breaking from the masterpiece approach. From the perspective of society museums and more specifically that of the Musée de la ville de SaintQuentin-en-Yvelines. In fact because the museum considers publics as actors who participate in the elaboration of the discourse, because their position in the community invites them to question rather than assert, they in fact play a mediation role around participation.

Mediation holds a privileged place in these institutions. The approach of the Musée de la ville nouvelle de Saint-Quentin-en-Yvelines, dedicated to a recent heritage not readily obvious to the inhabitants, is also based on the participation of local actors in order to build its discourse on the city. Its continuously renewed museographic mechanism questions and demolishes the given ideas on the city. Its cultural action binds with the visitors' viewpoints to construct a dialogue remote from the "lesson on architecture." Finally, the museum questions the event-driven rise to power and its impact on public democratization.

To conclude, museums have developed these last years great fields of contemplation directed at their publics that go beyond contemplation and education missions. Numerous approaches are still worthy of being experimented and evaluated in order to renew the image and impact of the museum. 